\title{
Lack of effect of warfarin on the restenosis rate or on clinical outcome after balloon coronary angioplasty
}

\author{
P URBAN, N BULLER, K FOX, L SHAPIRO, J BAYLISS, A RICKARDS
}

From the National Heart Hospital, London

SUMMARY Between September 1985 and April 1987, 110 consecutive patients who had successful coronary angioplasty were included in a randomised prospective controlled evaluation of the effects of warfarin on restenosis. The warfarin $(n=56)$ and the control $(n=54)$ groups were not different in terms of age, sex, previous coronary bypass surgery or coronary balloon angioplasty, severity of symptoms, and frequency of multivessel disease or of total coronary occlusions. Warfarin was started on the day of the procedure and the dosage was adjusted to maintain the thromboplastin international normalised ratio $\geqslant 2 \cdot 5$. One hundred and five $(96 \%)$ of the patients were given verapamil and other antianginal drugs were prescribed as needed. Low molecular weight dextran and heparin were given during the procedure and heparin was continued for 24 hours in all patients. One hundred and eight $(98 \%)$ of patients were followed up clinically after a median of five months (range 1-20). Eighty five (77\%) had follow up angiography at five months. In the warfarin group symptoms improved in $46(85 \%)$ patients by at least 1 angina class and 31 $(57 \%)$ were symptom free; the exercise test remained positive in $20(36 \%)$ patients and the angiographic restenosis rate was $25 \%$ per lesion and $29 \%$ per patient. There were no major bleeding complications. In the control group $46(85 \%)$ patients were improved by at least 1 angina class and $31(57 \%)$ were symptom free; the exercise test was positive in $11(21 \%)$ patients and the angiographic restenosis rate was 33\% per lesion and $37 \%$ per patient. Although the incidence of angiographic restenosis tended to be lower with warfarin, none of these differences was significant.

These data suggest that the combination of verapamil and warfarin, in the absence of aspirin, is not significantly better than verapamil alone in preventing symptom recurrence or angiographic restenosis after coronary angioplasty.

Restenosis, usually occurring during the first three to six months after coronary balloon angioplasty, remains one of the main limitations of the technique. ${ }^{12}$ Although several risk factors associated with an increased rate of recurrence have been identified, ${ }^{134}$ our understanding of the underlying pathophysiological mechanisms remains incomplete, and no drug regimen has yet been shown consistently to affect the occurrence of restenosis. ${ }^{5-7}$

As it is currently understood, restenosis is a response to balloon induced arterial injury and is determined by multiple elements. Mechanical factors (elastic recoil of the dilated arterial segment, medial dissection, and intimal flap formation),

Requests for reprints to Dr A Rickards, National Heart Hospital, Westmoreland Street, London W1M 8BA.

Accepted for publication 11 August 1988 haemostatic factors (thrombus formation and platelet deposition), and fibromuscular proliferation all occur and interact to induce restenosis. ${ }^{8-10}$

There is good experimental and clinical evidence that early local thrombus formation is a key element in restenosis. ${ }^{11} 12$ Because of these data, and despite a previously reported negative result, ${ }^{13}$ we undertook a prospective randomised controlled study of the effects of warfarin on the clinical outcome and angiographic restenosis rate after angioplasty.

\section{Patients and methods}

PATIENTS AND MEDICATION

Between September 1985 and April 1987, 155 consecutive patients underwent coronary artery balloon angioplasty at our hospital. The initial success rate was $76 \%$ for 140 stenoses and $47 \%$ for 15 occlusions-113 patients had a successful and 
uncomplicated procedure (target lesion(s) with < $50 \%$ residual stenosis). One hundred and ten of these patients were randomly assigned to receive either warfarin and verapamil or verapamil alone after the procedure. Table 1 gives the baseline clinical data of both groups. Only lesions causing $\geqslant \mathbf{7 0} \%$ stenosis were considered for angioplasty. All types of angioplasty were included-that is stenosis or occlusion, single or multivessel procedures, native vessels or vein grafts, primary stenosis or restenotic lesions (table 2). Twelve patients were included twice and two patients were included three times. During the study period, three patients were excluded before randomisation (one with a recent cerebrovascular accident that contraindicated the use of anticoagulants, one taking long term warfarin because of atrial fibrillation, and one who was successfully treated with streptokinase for recurrent chest pain with ST segment elevation a few hours after angioplasty).

All patients were given heparin 10000 IU intravenously before angioplasty together with an infusion of low molecular weight dextran. Heparin was continued for 24 hours after the procedure, until the arterial sheath was taken out. Verapamil was started on the day of the procedure. In the anticoagulated group warfarin was begun on the evening of the angioplasty day. Dosage was adjusted to obtain a thromboplastin international normalised ratio of $\geqslant 2 \cdot 5 .{ }^{14}$ Verapamil and warfarin were continued either for six months or until a follow up angiogram was obtained. No antiplatelet agents were prescribed at discharge, but one patient in the warfarin group and six patients in the control group were given aspirin at some time during the follow up period. Aditional antianginal treatment such as nitrates or $\beta$ blockers were given as required. There was no difference in the postoperative management

Table 1 Clinical and angiographic data for warfarin and control groups

\begin{tabular}{lll}
\hline Data & $\begin{array}{c}\text { Warfarin } \\
(n=56)\end{array}$ & $\begin{array}{l}\text { Control } \\
(n=54)\end{array}$ \\
\hline Number & 56 & 54 \\
Mean age (SD) & $56(9)$ & $57(10)$ \\
Sex (\% male) & 88 & 83 \\
CCS class 3 or 4 & $37(66)$ & $42(77)$ \\
Unstable angina & $6(11)$ & $13(24)$ \\
Previous AMI & $22(39)$ & $18(33)$ \\
Previous CABG & $7(13)$ & $11(20)$ \\
Previous PTCA & $13(23)$ & $9(17)$ \\
Positive stress test & $39 / 44(89)$ & $32 / 39(82)$ \\
Multivessel disease & $26(46)$ & $27(50)$ \\
\hline
\end{tabular}

Except where indicated numbers in parentheses are percentages. CCS, Canadian Cardiovascular Society; unstable angina, rest pain together with transient electrocardiographic changes; AMI, acute myocardial infarction; CABG, coronary artery bypass grafting; PTCA, percutaneous transluminal coronary angioplasty.
Table 2 Angioplasty data for warfarin and control groups $\stackrel{.}{.}$

\begin{tabular}{|c|c|c|}
\hline & $\begin{array}{l}\text { Warfarin } \\
(n=56)\end{array}$ & $\begin{array}{l}\text { Control } \\
(n=54)\end{array}$ \\
\hline 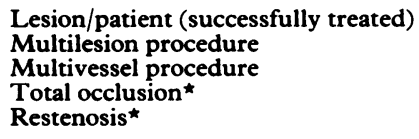 & $\begin{array}{l}1 \cdot 2 \\
10(18) \\
3(5) \\
4(7) \\
6(11)\end{array}$ & $\begin{array}{l}1 \cdot 3 \\
10(19) \\
4(7) \\
2(4) \\
9(17)\end{array}$ \\
\hline $\begin{array}{c}\text { Target: } \\
\text { LMS } \\
\text { LAD } \\
\text { LCX } \\
\text { RCA } \\
\text { VG }\end{array}$ & $\begin{array}{l}0 \\
33(57) \\
7(12) \\
13(22) \\
5(9)\end{array}$ & $\begin{aligned} & 1(2) \\
& 32(57) \\
& 6(11) \\
& 14(25) \\
& 3(5)\end{aligned}$ \\
\hline
\end{tabular}

*Present in at least one of the target lesions for a given procedure. Numbers in parentheses are percentages.

LMS, left main stem coronary artery; $L A D$, left anterior descending $\vec{P}$ coronary artery; LCX, left circumflex coronary artery; RCA, righter coronary artery; VG, vein graft.

of patients undergoing angioplasty of single or multiple lesions.

\section{FOLLOW UP}

Most patients were followed up as outpatients at the National Heart Hospital. For those who were unableo to return, information was obtained from the referring physician whenever possible. Anticoagulationo was monitored in anticoagulant clinics with regulars checks of the international normalised ratio. Clinicalo follow up data, stress testing, and follow upo angiograms were generally obtained three to nineo months after angioplasty, or earlier if symptoms $\overrightarrow{\vec{\sigma}}$ recurred or became worse.

\section{END POINTS OF STUDY}

Clinical state, the response of the ST segment to exercise, and the angiographic appearance of theo angioplasty site were analysed at follow up. Before $-\overline{-}$ angioplasty and at follow up we used the Canadian 3 . Cardiovascular Society (CCS) grading system for subjective assessment of anginal symptoms. Unstable angina was defined as rest pain together witho reversible electrocardiographic changes. The exercise test was symptom limited and done according too the modified treadmill Bruce protocol with monitoring of 12 electrocardiogram leads. ST segmento depression of $\geqslant 1 \mathrm{~mm}$ developing in at least one lead 0 during or after exercise was regarded as a positive $\mathbb{W}^{-}$ test. Angiographic restenosis was considered to beo present when there was $\geqslant 50 \%$ reduction in luminal diameter estimated from at least two projections atc the site of the previous angioplasty.

We compared the results in the warfarin and the control groups by Student's $t$ test for continuouso variables and by the $\chi^{2}$ test for discrete variables. $\overparen{D}$ Differences of $p<0.05$ were considered to bed statistically significant. 
We calculated that for a power of 0.75 and a significance level of 0.05 , we would need 54 patients in each group to show a $50 \%$ reduction in the restenosis rate.

\section{Results}

The two groups (warfarin and control) were similar for all the clinical and procedural variables tested (tables 1 and 2).

\section{CLINICAL OUTCOME}

At follow up we had subjective information on anginal symptoms for $108(98 \%)$ of 110 patients $(96 \%$ of the warfarin group and $100 \%$ of the control group). In the warfarin group (median follow up six months, range $1-18$ months), $46(85 \%)$ of 54 patients had improved by $\geqslant 1$ Canadian Cardiovascular Society angina class, and $31(57 \%)$ were symptom free. Two patients $(4 \%)$ were in class 4 and no patient had unstable angina. There were no major bleeding complications in the warfarin group. The results were similar for the control group (median follow up five months, range $1-20$ months) where $46(85 \%)$ of 54 were improved and $31(57 \%)$ were symptom free. One patient ( $2 \%$ ) was in class 4 and two had unstable angina $(4 \%)$.

\section{STRESS TESTING}

Results of stress testing at follow up were available for $81(74 \%)$ of 110 patients $(75 \%$ of the warfarin group and $72 \%$ of the control group). In the warfarin group, $15(36 \%)$ of 42 patients had an appreciable depression of the ST segment with exercise, and in 16 $(38 \%)$ of 42 chest pain developed. The test was symptom limited and patients reached a median of Bruce protocol stage 3 (range 1-5). In the control group, eight $(21 \%)$ of 39 patients had significant ST segment depression with exercise and in $12(31 \%)$ chest pain developed. They reached a median of stage 3 of the Bruce protocol (range 0-5). None of these differences was statistically significant.

\section{ANGIOGRAPHY}

Angiographic follow up was obtained in $85(77 \%)$ of 110 patients $(75 \%$ of the warfarin group and $80 \%$ of the control group). After a median follow up of five months (range 1-19 months) in the warfarin group the restenosis rate was $29 \%$ per patient $(12$ of 42$)$ and $25 \%$ per lesion ( 12 of 49 ). In the control group, the median follow up was five months (range 1-20 months), the restenosis rate per patient was $37 \%$ (16 of 43 ), and per lesion $33 \%$ (18 of 54 ).

During the follow up period in the warfarin group eight $(15 \%)$ patients underwent a repeat angioplasty and two $(4 \%)$ had elective coronary artery bypass grafting. Corresponding figures in the control group were nine $(17 \%)$ and one $(2 \%)$ respectively. There were no deaths in either group during the follow up period.

\section{Discussion}

Despite our initial hypothesis that warfarin would favourably affect clinical and angiographic outcome after coronary balloon angioplasty, we found that our results confirmed a previous finding ${ }^{13}$ and did not show that oral anticoagulation had a significant effect on medium term results. This is the first comparison of the effects of anticoagulation with a control group that essentially had no antiplatelet treatment. Angiography showed that there was a trend towards a reduction in the restenosis rate for the patients in the warfarin group, and it could be argued that a larger study population might have shown a significant difference or have retrospectively identifed a subset in whom warfarin reduced the incidence of restenosis. However, in the absence of any difference in the subjective evaluation of anginal symptoms or electrocardiographic changes during stress testing, such an angiographic trend is difficult to interpret. The angiograms were analysed according to current clinical practice and the percentage reduction in the diameter at the angioplasty site was estimated visually. The angiographic views were standarised as far as possible and the films interpreted without knowledge of the individual's clinical condition. The cut off value of $50 \%$ taken to represent significant restenosis is widely accepted ${ }^{10}$ but arbitrary. Our study was limited by the lack of quantitative computer assisted analysis of the angioplasty site. ${ }^{15}$

Compliance with the anticoagulation regimen after angioplasty has been reported to be less than ideal. ${ }^{13}$ Because the patients in this series were regularly followed in anticoagulant clinics, compliance with treatment was good.

The timing of anticoagulation treatment in relation to angioplasty is probably an important factor, and in the present series there was a gap of 24-48 hours between the end of heparin administration after angioplasty and the establishment of effective oral anticoagulation. In view of the currently available data ${ }^{112}$ such an interruption is probably undesirable, since the thrombosis and platelet deposition that contribute to restenosis may well occur at a very early stage. At the time when the study was designed it was thought that withdrawing the arterial sheath under full anticoagulation would cause problems, but experience with patients undergoing intracoronary stenting ${ }^{16}$ has shown that it is feasible with few complications.

The best drug regimen for preventing restenosis 
after angioplasty remains to be determined. Thornton et al reported that warfarin was no better than aspirin, and showed that aspirin was better than warfarin for patients with a long history of angina. ${ }^{13}$ Fleck et al showed that interruption of aspirin treatment during follow up was associated with an increased risk of restenosis, ${ }^{17}$ but their conclusions have been challenged by others. ${ }^{10}$ Calcium channel blockers are widely prescribed after angioplasty, but currently there are no data to support this practice. ${ }^{56}$

Aspirin together with dipyridamole has recently been shown to reduce occurrence of acute closure after angioplasty, ${ }^{18}$ and a similar combination is also known to be effective in maintaining patency in saphenous vein grafts. ${ }^{19}$ Antiplatelet agents are currently being evaluated for their ability to prevent restenosis in several continuing controlled clinical trials. $^{7}$

The effect of individual compounds on restenosis should be tested first. But the success of combination treatments with oral anticoagulants and one or several antiplatelet agents together may prove to be more important if the current interest in intravascular stenting devices ${ }^{16}$ is maintained. This is because the initial thrombogenic properties of the currently used metallic stents means that empirical vigorous anticoagulation together with antiplatelet medication has been used during the first months after implantation. ${ }^{1620}$ If the restenosis rate is lower that that expected with conventional balloon angioplasty, the benefits derived from the mechanical support and those obtained by the drug regimen alone will have to be determined.

\section{References}

1 Meier B. Restenosis after coronary angioplasty. Eur Heart J 1988;9:c:1-6.

2 Myler RK, Topol EJ, Shaw RE, et al. Multiple vessel coronary angioplasty; classification, results, and patterns of restenosis in 494 consecutive patients. Cathet Cardiovasc Diagn 1987;13:1-15.

3 Douglas JS, King SB, Roubin GS. Influence of methodology of percutaneous transluminal coronary angioplasty on restenosis. Am J Cardiol 1987;60:29B33B.

4 Urban P, Meier B, Finci L, de Bruyne B, Steffenino G, Rutishauser $W$. Coronary wedge pressure: a predictor of restenosis after coronary balloon angioplasty. $\mathrm{J} \mathrm{Am}$ Coll Cardiol 1987;10:504-9.
5 Whitworth HB, Roubin GS, Hollman J, et al. Effect of nifedipine on recurrent stenosis after percutaneous transluminal coronary angioplasty. J Am Coll Cardiof 1986;8:1271-6.

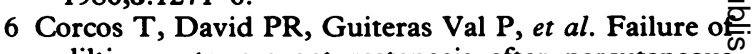
diltiazem to prevent restenosis after percutaneouss transluminal angioplasty. Am Heart J 1985;109 926-31.

7 Blackshear JL, O'Callaghan WG, Califf RM. Medica $\mathcal{L}^{\circ}$ approaches to prevention of restenosis after coronary angioplasty. J Am Coll Cardiol 1987;9:834-48.

8 Faxon DP, Sanborn TA, Weber VJ, et al. Restenosis following transluminal angioplasty in experimenta 5 atherosclerosis. Arteriosclerosis 1984;4:189-95.

9 Bettmann MA. Anticoagulation and restenosis afters percutaneous transluminal coronary angioplasty. Amp $J$ Cardiol 1987;60:17B-9B.

10 Meier B. Coronary angioplasty. Orlando: Grune andr Stratton, 1987.

11 Fuster V, Adams PC, Badimon JJ, Chesebro JH? Platelet-inhibitor drugs' role in coronary artery dis ease. Prog Cardiovasc Dis 1987;29:325-46.

12 Harker LA. Role of platelets and thrombosis in mechan $\frac{\mathbb{N}}{3}$ isms of acute occlusion and restenosis after angio plasty. Am J Cardiol 1987;60:20B-8B.

13 Thornton MA, Gruentzig AR, Hollman J, King SB. Douglas JS. Coumadin and aspirin in prevention of recurrence after transluminal coronary angioplasty: $\dot{q}$ randomized study. Circulation 1984;69:721-7.

14 Wessler S, Gitel SN. Pharmacology of heparin and warfarin. J Am Coll Cardiol 1986;8:10B-20B.

15 Reiber JHC, Serruys PW, Koijman CJ, et al. Assess ment of short-, medium-, and long-term variations inf arterial dimensions from computer-assisted quanti $\underset{\overrightarrow{2}}{\stackrel{2}{ }}$ fication of coronary cineangiograms. Circulation 1985;71:280-8.

16 Sigwart U, Puel J, Mirkovitch V, Joffre F, Kappenber ger L. Intravascular stents to prevent occlusion and restenosis after transluminal angioplasty. $N$ Engl Med 1987;316:701-6.

17 Fleck E, Dirschinger J, Rudolph W. Quantitative Koronarangiographie vor und nach PTCA. Herz3 1985;10:313-20.

18 Barnathan ES, Schwartz JS, Taylor L, et al. Aspiriñ and dipyridamole in the prevention of acute coronary thrombosis complicating coronary angioplasty. Cir윽 culation 1987;76:125-34.

19 Chesebro JH, Clements IP, Fuster V, et al. A platelets inhibitor drug trial in coronary artery bypass opera $\overline{\bar{N}}$ tions. Benefit of perioperative dipyridamole and aspirin therapy on early post-operative vein graft patency. N Engl J Med 1982;307:73-8.

20 Sigwart U, Kaufmann U, Goy JJ, et al. Prevention of coronary restenosis by stenting. Eur Heart $J$ 1988;9울 c:31-7. 